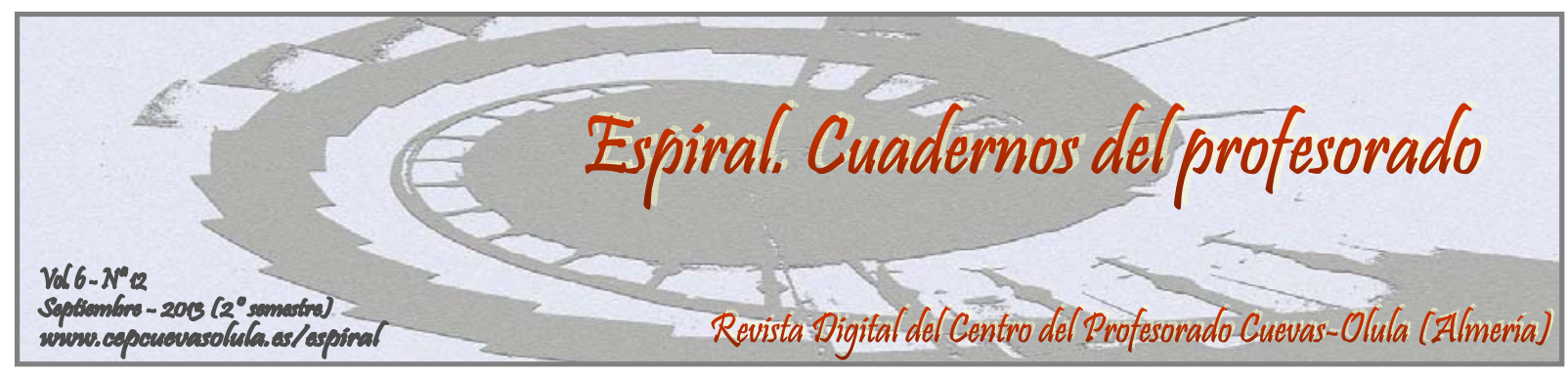

\title{
LA EDUCACIÓN AUDITIVA COMO EJE VERTEBRADOR DE LA EDUCACIÓN MUSICAL EN LA EDUCACIÓN SECUNDARIA OBLIGATORIA
}

\section{HEARING EDUCATION AS THE BACKBONE OF THE MUSICAL EDUCATION IN COMPULSORY SECONDARY EDUCATION}

\author{
Ana María Botella Nicolás ${ }^{(1)}$ y José Vicente Gimeno Romero ${ }^{(2)}$
}

(1) Departamento de Didáctica de la Expresión Musical, Plástica y Corporal de la Universidad de Valencia, Universidad de Valencia, España

(2) IES L'Eliana de Valencia, España

RESUMEN: El presente trabajo se enmarca dentro de un estudio de mayores dimensiones motivado por el interés de los autores en la didáctica de la audición musical, convencidos de que los principios fundamentales sobre los que se debería apoyar la educación musical actualmente en la Educación Secundaria Obligatoria son, por una parte, la necesidad de una mayor contribución de la misma a una educación integradora de todas las dimensiones del ser humano, y por otra, la conveniencia de otorgarle un mayor protagonismo a la educación auditiva en el proceso de enseñanza-aprendizaje de la materia de música en dicha etapa educativa. El propósito es dar respuesta a una serie de interrogantes que surgen en torno a las estrategias metodológicas más adecuadas para llevar a cabo una verdadera educación auditiva, para lo cual se ha utilizado el método de la Action Research, un enfoque cualitativo cuyo objeto de estudio es la propia práctica educativa, con la intención de mejorarla, desde la convicción de que únicamente a través de la interacción directa y la participación en la experiencia real el investigador puede obtener conocimientos auténticos. Los resultados obtenidos con la experiencia didáctica llevada a cabo aportan una serie de sugerencias que deberían ser tenidas en cuenta para la práctica docente del profesorado de música.

Palabras clave: Educación integral, percepción musical, didáctica de la audición musical.

ABSTRACT: This work is part of a larger study, motivated by the interest of the authors in the didactics of musical hearing, convinced that the fundamental principles on which musical education should support currently in the secondary education are, on one hand, the need for a greater contribution of musical education to an education including all human being dimensions, and on the other hand, the convenience of giving a greater role to the auditorial education in the process of teaching and learning music, which is what matters in this educational stage. The purpose is to answer a series of questions that arise around the approaches best suited to carry out a real education hearing, for which we used the method of the Action Research, a qualitative approach whose object of study is the own educational practice, with the intention of improving it, from the conviction that only through direct interaction and participation in the actual experience the researcher can get authentic knowledge. The results obtained with the accomplished teaching experience with a range of suggestions that should be taken into account in the teaching practice of teachers of music.

Key words: Integral education, musical perception, didactical of musical hearing. 
Botella Nicolás, A. M., y Gimeno Romero, J. V. (2013). La educación auditiva como eje vertebrador de la educación musical en la Educación Secundaria Obligatoria. Espiral. Cuadernos del Profesorado, 6(12), 61-70. Disponible en: http://www.cepcuevasolula.es/espiral.

Fecha de recepción: 06/05/2013

Fecha de aceptación: 02/09/2013
Enviar correspondencia a: ana.maria.botella@uv.es

\section{1.- INTRODUCCIÓN}

La música está más presente que nunca en la vida diaria de los jóvenes que cursan los distintos niveles de la Educación Secundaria Obligatoria. Es evidente que la mayoría de ellos escucha música a diario. Sin embargo, a pesar de vivir rodeados de ella, en el contexto de la educación formal, se sigue infravalorando lo que ésta puede aportar a su formación.

La educación musical que los jóvenes deben recibir en el contexto de la enseñanza obligatoria debe abordar una auténtica "sensibilización” de los mismos hacia todo tipo de música, preparándolos para poder elegir libremente sus preferencias musicales, y fomentado en ellos el "espíritu crítico”. Es necesario replantearnos el papel que debe desempeñar la audición en la educación musical así como la manera de llevar a cabo dicha educación auditiva.

La pedagogía actual viene a confirmar, desde perspectivas rigurosamente científicas, la necesidad de diseñar modelos educativos que potencien todas las facetas del ser humano, siendo muchos los autores que han fundamentado la importancia de la educación musical en la formación integral de los alumnos. Autores como Leiva y Mates (1998) o Ramírez (2006) entre otros, coinciden en destacar la gran diversidad de emociones, sentimientos y afectos que se experimentan al vivir la experiencia musical, lo que sin duda favorece en los alumnos la confianza en sí mismos, el respeto a su persona y a los demás, el desarrollo del espíritu crítico, etc., componentes esenciales en la concepción de una educación verdaderamente integral, que supere el enfoque de la educación meramente constructivo-cognitivista, que es en realidad el fin último de la educación.

La educación limitada exclusivamente al intelecto se ha demostrado insuficiente en algunos aspectos, consiguiéndose mejores resultados en el proceso de enseñanza-aprendizaje cuando se abordan, además, las dimensiones afectiva y de relación interpersonal. Tanto es así, que podríamos considerar que la enseñanza que no tiene en cuenta la inteligencia de las emociones y los sentimientos corre el peligro de educar sólo a una parte de la persona, pero no al alumno de una manera integral (Leiva y Mates, 1998; Campbell, 1998; Ramírez, 2006) y es en este contexto donde queremos situar la educación musical que deben recibir los jóvenes que cursan esta etapa.

Gardner (1995) afirma en su teoría de las Inteligencias Múltiples, que la música estimula la totalidad de las inteligencias con las que experimentamos y comprendemos el mundo, introduciendo el concepto de inteligencia intrapersonal, entendida ésta como el conocimiento de los aspectos internos de uno mismo, el acceso a la vida emocional, a los propios sentimientos, así como la capacidad de distinguir las emociones, y ponerles un nombre.

En este sentido, las principales corrientes pedagógico-musicales surgidas a lo largo del siglo XX, han demostrado que la educación auditiva es un vehículo idóneo para desarrollar la atención, la capacidad de concentración, la memoria y la sensibilidad, contribuyendo así al desarrollo intelectual, afectivo e interpersonal de los jóvenes.

Dicha educación auditiva, debe empezar por el desarrollo de la capacidad de escuchar. Palacios (1997) dice al respecto que: "escuchar es la palabra clave (...) la música solo existe si hay atención” (p. 33). La finalidad debe ser que el alumno escuche más y mejor, pues a escuchar -y a dejar escuchar-, se aprende escuchando. Pero las personas no pueden apreciar el valor de las cosas sin antes comprenderlas: "sólo puede llegar a gustar lo que se conoce" (Palacios, 2011, p. 52), y es aquí donde surge la necesidad de una verdadera educación auditiva, que proporcione a los alumnos los conocimientos necesarios para ampliar su capacidad de apreciación, y en consecuencia, de comprensión, de la música escuchada. 
Del currículo de la Educación Secundaria Obligatoria (Decreto 112/2007, p. 30568), se desprende la necesidad de ofrecer a los alumnos una educación auditiva que aborde la sensibilización de éstos hacia la música, tendiendo puentes entre su mundo y la música que se les ofrece en el aula con el fin de que puedan elegir libremente sus preferencias musicales-. En este sentido, la enseñanza de la materia de música debe:

Potenciar las capacidades de escuchar, reconocer y retener las relaciones que configuran las estructuras musicales y por ello mismo, ha de orientar en la diversificación de gustos musicales y en la formación de un espíritu crítico (...). Hay músicas de gran riqueza, pero sólo unas y unos pocos pueden o saben disfrutarlas debido a que el gran público no está capacitado para percibir la gran cantidad de parámetros musicales que se ofrecen. Se trataría pues de un problema de educación. No es que les guste o no les guste, sino que no tienen las herramientas en sus oídos y en su entendimiento para emitir un juicio de gusto.

Se trata, como afirma Paynter (1999) de "ayudar a nuestros alumnos a beneficiarse de algo más que un contacto pasajero con el arte de la música" (p. 13), tomando como punto de partida la diferenciación que establece Edgar Willems (2011) entre “oír, escuchar y entender” (p. 49).

Este pedagogo identifica oír con la sensorialidad auditiva -que supone únicamente tener un sistema auditivo operativo-; escuchar, con la afectividad auditiva -que implica tener la intención de oír algo de manera preferente sobre el resto de estímulos sonoros que nos rodean-; y finalmente, entender, con la inteligencia auditiva -lo que conlleva hacer voluntariamente una elección de aquello que interesa-.

Así, la educación auditiva debe enseñar al alumno a escuchar música y a reflexionar sobre lo percibido, con la finalidad de llegar a entenderla, porque éstos perciben muchas más cosas de las que entienden.

Además, se debe hacer de una forma creativa, pues aunque se trate de una clase de creatividad distinta a la que se pueda dar en el hecho de interpretar, la audición también exige un importante esfuerzo de imaginación, (Palacios, 1997, p. 154):

Escuchar es una actividad, no una pasividad. Los oyentes, por el mero hecho de escuchar, participamos de una manera dinámica. La audición no sólo es exterior, es también interior. Para escuchar debemos intervenir activamente y no quedarnos como simples receptores de sonidos: tenemos que poner de nuestra parte, atender, vencer la vaguería.

Es necesario pues, fomentar la predisposición a escuchar, sobre todo para superar la resistencia que los alumnos puedan ofrecer ante la audición de cualquier género musical que no se corresponda con sus gustos personales, aún a riesgo de que en su lugar aparezca otro tipo de rechazo, el de su elección personal, pero ahora ya, libre y consciente.

Por otra parte, hay que tener en cuenta que sus actitudes hacia la audición musical pueden verse influenciadas tanto por el entorno acústico que les rodea, como por el contexto social en el que se desenvuelven. Los alumnos viven inmersos en un ambiente muy saturado de estímulos auditivos, lo que está creando un paisaje sonoro altamente contaminado, que produce una cierta tendencia a la dispersión mental. El concepto de paisaje sonoro fue utilizado por primera vez por el compositor y pedagogo canadiense Raymond Murray Schafer (1933) en su libro El nuevo paisaje sonoro, publicado en 1969 por la editorial Ricordi Americana para referirse al entorno sonoro concreto de un lugar determinado.

Vivir rodeados de esta polución sonora desgasta la sensibilidad auditiva de los alumnos, los cuales están tan acostumbrados a hablar y trabajar rodeados de un ambiente musicalizado, que cuando es el momento de escuchar de verdad, con todas las implicaciones intelectuales que ello supone, dicha acción les exige un esfuerzo de concentración cada vez mayor. 
En cuanto a las influencias provenientes del entorno social, hay que destacar lo que Josefa Lacárcel (2003) denomina modas y prejuicios, cuando habla de las preferencias musicales de los jóvenes (p. 13):

Pertenecemos al contexto histórico en el que vivimos, y esto hace que seamos miembros de pequeñas células sociales que a su vez van insertándose en unidades sociales cada vez más amplias y masivas, formando el tejido social con toda la complejidad de pertenencias, comportamientos y relaciones.

Así, entramos en el campo de investigación de la Psicología Social de la música, la cual trata de dilucidar la manera en que las fuerzas sociales influyen en los gustos musicales de los alumnos, y establecen ciertos prejuicios a determinados estilos musicales.

La necesidad de otorgarle un mayor protagonismo a la educación auditiva en el proceso de enseñanza-aprendizaje de la música en la educación con carácter obligatorio, se basa, por una parte en la observación de la realidad escolar en la que vivimos actualmente y la necesidad de que los docentes nos adaptemos a los constantes cambios que se dan en la misma, y por otra, en el hecho contrastado de que es desde la audición desde donde podemos conseguir un verdadero acercamiento a sus intereses y motivaciones.

El currículo de la Educación Secundaria Obligatoria antes mencionado ya nos advierte de que "la consecución de los objetivos de la educación no puede hacerse sin considerar las diferentes actitudes y necesidades de los jóvenes a esas edades, así como su variedad de intereses” (p. 30403). La realidad escolar a la que aludíamos anteriormente nos demuestra que la música está más presente que nunca en la vida diaria de los jóvenes que cursan los distintos niveles de la Educación Secundaria Obligatoria, pero la música que estos escuchan no se corresponde con la que en la mayoría de los casos se les ofrece desde el ámbito de la educación formal, lo cual conlleva a un cuestionamiento del valor educativo que se le atribuye a la misma.

\section{2.- APROXIMACIÓN CONCEPTUAL AL TÉRMINO AUDICIÓN MUSICAL}

Etimológicamente el término audición musical viene del latín audito, que es la acción de audire (oír), es decir, percibir a través del oído los sonidos o lo que es lo mismo, el proceso por el cual el oído trata de construir la unidad de la obra al hilo del tiempo. Para Barceló (1998, p. 13), la audición es:

La forma en que el organismo humano interactúa con el conjunto de estímulos y estructuras musicales; se ve materializada por tres características (...): 1) el control y la coordinación sensorial (...) que su práctica precisa; 2) el desarrollo característico de sus elementos en el tiempo; 3) el contenido eminentemente abstracto y sintáctico que transporta per se, basado en las interrelaciones entre las cualidades del sonido y las diversas leyes de construcción del discurso musical.

Si partimos del hecho de que la música es sonido, es obvio e innecesario el pretender poner en evidencia la importancia de la audición en el proceso de la formación musical del alumno. Ésta puede utilizarse como técnica para favorecer el desarrollo de la atención, el hábito de escuchar, la observación, el análisis y el desarrollo de la sensibilidad. Además la audición implica también un trabajo analítico y crítico

Entre las características especiales de la experiencia musical, la escucha creativa se perfila como uno de los factores clave, no sólo en la invención y la interpretación de la música, sino también en la apreciación del aficionado que asiste a conciertos o escucha discos. En palabras de Maneveau (1993, p. 268):

Hay que enseñar y aprender a escuchar para oír y entender la música, pero es también enseñando a escucharla y entenderla como se cultivan las capacidades de escucha en general (...). Enseñar a escuchar es una tarea que sobrepasa la finalidad artística para situarse a un nivel de una ampliación y extensión de las relaciones humanas. Oír música es en primer lugar oír al mundo, es decir, oír y escuchar al otro. 
Enseñar a escuchar plenamente la música puede llevar a una mejor comunicación con nuestros semejantes.

Bajo el término audición -íntimamente relacionado con el de educación auditiva-, se define uno de los procedimientos más característicos e importantes de la educación musical, ya que a partir de él se van generando la mayoría de actividades y contenidos musicales. Cabe señalar como objetivos específicos de la educación auditiva:

- Percibir la música.

- Diferenciar acontecimientos sonoros.

- Cultivar el gusto por la música.

Para Malbrán (1996, 67-68), son seis los atributos críticos de la audición musical:

- La audición musical es relacional: se basa en el establecimiento de vinculaciones significativas del discurso musical a las que se accede desde la experimentación concreta con la materia musical y sonora.

- La audición musical es sincrónica: requiere simultáneamente prestar atención al discurso musical, configurar una representación acerca de lo que se está escuchando, y seguir escuchando al mismo tiempo.

- La audición musical es reversible: opera en el tiempo con imágenes en permanentes idas y vueltas en tiempo real, integrando lo que se escucha en el momento con lo inmediato anterior.

- La audición musical es predictiva: nuestra mente tiende a anticipar cómo va a seguir el discurso musical. La confirmación de las hipótesis así generadas o el reemplazo por otras, es un rasgo distintivo de los buenos oyentes.

- La audición musical es acumulativa: utiliza patrones melódicos, rítmicos y armónicos producto de aprendizajes previos.

- La audición musical es jerárquica: en el lenguaje musical procede de la estructura superficial a la profunda.

La audición musical es una actividad metacomponencial que pone en juego habilidades y disposiciones de alto orden que integran la reflexión, la actitud significativa, el pensamiento constructivo y la persistencia de la atención. Y escuchar es encontrar sentido a las relaciones sonoras presentes en una obra musical.

Resumiendo, y citando de nuevo a Malbrán (1996, p. 68), podemos decir que la audición musical puede entenderse "como un modelo múltiple y simultáneo de procesamiento de la información ya que requiere escuchar, formar una representación mental de lo que se está escuchando, predecir cómo va a continuar el discurso musical y comparar con lo que realmente ocurre mientras se escucha".

\section{3.- OBJETIVOS}

Iniciada ya la segunda década del siglo XXI, como docentes de música y conocedores de la realidad que se vive en las aulas, son varias las cuestiones que nos planteamos al respecto: ¿cómo adaptar a la nueva realidad escolar las estrategias didácticas para llevar a cabo la educación auditiva que pretendemos?, ¿cómo establecer puntos de contacto entre la música que les ofrecemos en las aulas y la que ellos escuchan?, ¿cómo influye la elección del repertorio a trabajar en las aulas en la motivación que muestran los alumnos hacia las actividades de audición?, ¿cómo actualizar el repertorio de música culta a utilizar en las clases?, etc.

Para dar respuestas a las preguntas planteadas, hemos llevado a cabo un trabajo en el que se ha combinado la investigación y la innovación en el aula, y que se ha estructurado en torno a cuatro objetivos:

- Apreciar la importancia que tiene el desarrollo de las capacidades perceptivas en la formación integral de los alumnos. 
- Elaborar una selección de obras de música culta idóneas en términos didácticos para trabajarlas en el aula.

- Establecer paralelismos entre obras de música culta y músicas que respondan a las preferencias del alumnado.

- Comprobar en qué medida la elección del repertorio influye en las actitudes, con que los alumnos, afrontan las actividades.

\section{4.- METODOLOGÍA}

La metodología utilizada ha sido la Action Research, un enfoque cualitativo cuyo objeto de estudio es la propia práctica educativa, con la intención de mejorarla, desde la convicción de que únicamente a través de la interacción directa y la participación en la experiencia real el investigador puede obtener conocimientos auténticos. Como afirma Bresler "una de las principales diferencias entre la investigación-acción y otros enfoque cualitativos es su énfasis pragmático y orientado a la práctica como principal motivación para el investigador" (Díaz, 2006, p. 92).

Si bien en un principio la investigación-acción prolongó el paradigma cuantitativo, pasó a ser en mayoritariamente cualitativa en los proyectos iniciados por Stenhouse (1975) y Elliot (1991) desde finales de la década de 1970 hasta la actualidad.

Así, de los distintos enfoques cualitativos (etnografía, fenomenología, etc.) la investigaciónacción es el que se centra realmente en el ámbito "público" del aula -en el proceso de enseñanza y aprendizaje-, observando tanto la conducta del profesor como la de los estudiantes.

Por otra parte, pocos estudios son puramente cualitativos (Bresler y Stake, 1992). Además, para incrementar nuestra comprensión de la enseñanza, algunas veces puede ser útil utilizar mediciones cuantitativas tales como las encuestas y los cuestionarios.

Así, las fuentes de datos utilizadas han sido de una gran diversidad, con el fin de poder abordar las múltiples realidades presentes en el aula:

a) Un cuestionario aplicado a un grupo de 20 alumnos de $4^{\circ}$ de ESO, (cuyas edades están comprendidas entre los 15 y 17 años),

b) La grabación audiovisual de dos sesiones destinadas a discusiones en grupo -para su posterior análisis e interpretación-,

c) Entrevistas personales semiestructuradas entre el profesor investigador y cada uno de los 20 alumnos, $\mathrm{y}$

d) La observación participante y no participante, por parte del profesor y de una observadora externa que asistió a tres sesiones en el aula.

La experiencia se ha llevado a cabo en un Instituto de Enseñanza Secundaria de titularidad pública perteneciente a la red de centros docentes de la Comunidad Valenciana, cuyos alumnos son mayoritariamente de nacionalidad española, si bien hay algunos procedentes de países del Este (Bulgaria y Rumanía), Sudamérica (Ecuador Colombia), y Asia (China).

En primer lugar, se revisó la literatura existente sobre la influencia de la educación musical en la formación integral de los alumnos. A continuación, se pasó a los alumnos un cuestionario que elaboramos previamente con el fin de conocer sus hábitos de escucha, el cual nos aportó mucha información sobre la música que escuchan, la manera de escuchar, en qué situaciones lo hacen, en qué momentos del día, durante cuánto tiempo etc. Una vez elaborado el informe con los datos obtenidos se dedicó una sesión para discutirlos en grupo (sesión que fue grabada para poder analizar posteriormente con tranquilidad las intervenciones de los propios alumnos).

Posteriormente, siguiendo las indicaciones del propio currículo que señala que la presencia de la música en la etapa de Educación Secundaria Obligatoria debe considerar como punto de referencia, el gusto y las preferencias del alumnado, se realizaron 3 audiciones de canciones seleccionadas por los propios alumnos, lo cual, como indica Aranguren (2011), constituye una fuente de estrategias didácticas, siendo éstas las que siguen: 
- Waka Waka (Shakira)

- Al otro lado del río (Jorge Drexler)

- Snow (Red Hot Chili Peppers)

Para realizar dichas audiciones seguimos un modelo de análisis musical, susceptible de ser utilizado también con ejemplos de música culta, con el fin de utilizar una misma manera de trabajar para ambos tipos de música y que posibilitó el que los alumnos identificaran auditivamente las principales características de las diferentes dimensiones de la música. Este modelo incluía los siguientes parámetros:

a) Para un tema actual:

- Género: música de concierto, música para la escena, música para el audiovisual (cine, videoclip, publicidad, consolas), etc.

- Estilo: pop, rock, jazz, salsa, reggae, etc.

- Carácter: sensaciones que se desprenden de la música

- Formación Instrumental: bajo- guitarra- teclado- batería y voz, grupo pop, grupo rock, banda de jazz, teclados y sintetizadores electrónicos, instrumentos predominantes, alternancias y juego tímbrico, etc.

- Características: ¿predomina el aspecto rítmico o el melódico?

- Rasgos rítmicos: compás binario/ternario/cuaternario, ritmo muy marcado/ritmo fluido, células rítmicas que se repiten, ostinatos etc.

- Rasgos melódicos: melodía clara y limpia/ ornamentada, melodía fluida/discontinua, ámbito de la melodía (abierto/estrecho), dibujo de la línea melódica (plana/ondulada/ con grandes saltos)

- ¿El tempo es uniforme o hay cambios de tempo? si el tempo es uniforme ¿cómo lo definirías?

- Si hay cambios de tempo ¿̇son progresivos o repentinos? ¿en que sentido se producen?

- ¿ ¿La dinámica es uniforme o hay cambios? si es uniforme ¿cómo la definirías? Si hay cambios dinámicos ¿son progresivos o repentinos? ¿en qué sentido se producen?

- Armonía: tipo de acompañamiento, voces interiores, texturas,

- Si se trata de una obra vocal, idioma del texto, temática y contenido de la letra, relación música-texto.

- Forma y estructura: temas melódicos que aparecen, repeticiones de los temas, contrastes, etc.

- Entorno social y cultural: contextualización, entorno social, datos sobre el intérprete o grupo, valoración de su trayectoria, discos, aspectos publicitarios y promocionales, etc.

b) Para la música culta:

- Género: artística/funcional, vocal/instrumental, civil/religiosa, pura, descriptiva, para la escena, para el cine, para la publicidad, etc.

- Estilo: periodo y corriente artística.

- Carácter: sensaciones que se desprenden de la música, sentimientos que les sugiere.

- Formación Instrumental: orquesta sinfónica, orquesta de cuerda, grupo de cámara, solista, formación electroacústica, banda, coro, instrumentos predominantes, alternancias y juego tímbrico, etc. 
- Características: son comunes a las de la música actual.

- Forma y estructura: temas melódicos que aparecen, repeticiones de los temas, contrastes, etc.

- Entorno social y cultural: contexto histórico, situación del compositor en su entorno, la obra en la vida del compositor, aspectos extramusicales, etc.

Al mismo tiempo fuimos elaborando una selección de fragmentos de música culta que nos permitieran establecer similitudes con los aspectos del lenguaje musical trabajados en las canciones escuchadas anteriormente, y que por sus características resultaran idóneas en términos didácticos para cada caso, intentando además ampliar el repertorio de música culta que habitualmente aparece en los libros de texto, e incluyendo música culta de nuestros días. Finalmente las obras escuchadas fueron:

- Introducción y Augurios de primavera, de la primera Suite de La consagración de la primavera de Stravinsky, donde destacan cuestiones rítmicas, al igual que en la canción de Shakira.

- $\quad$ El Lied Ich will meine Seele tauchen, del ciclo Diechterliebe de Schumann, por su carácter íntimo, comparable al de la canción Al otro lado del río de Jorge Drexler.

- El Passacaglia de Ramón Ramos, donde a pesar de su apariencia contemporánea, se esconden recursos tan antiguos como el ostinato, al igual que en la canción Snow de Red Hot Chili Peppers.

Tras realizar las audiciones, se dedicó una sesión para discutir en grupo el tratamiento de algunas cuestiones referentes al lenguaje musical en un tipo de música y otro, la cual fue grabada para poder analizar posteriormente las intervenciones de los alumnos y hacer una interpretación de las mismas.

La observación participante por parte del profesor investigador del desarrollo de las clases en las que se trabajaron las audiciones, así como una entrevista personal de 20 minutos de duración con la totalidad de los 20 alumnos del grupo nos permitió comprobar en qué medida la elección del repertorio influye en las actitudes con que estos afrontan las actividades de audición, tanto en las clases como en sus casas cuando se les pide que realicen tareas.

Finalmente, las sugerencias hechas por la observadora externa, la cual asistió a tres sesiones (dos de audición, una de cada tipo de música, y la segunda sesión destinada a la discusión en grupo), aportaron a la investigación la objetividad necesaria para poder realizar cambios en la práctica educativa que realmente mejorasen el proceso de enseñanza-aprendizaje.

\section{5.- RESULTADOS}

En primer lugar queremos destacar el alto grado de aceptación de la experiencia realizada por parte de los alumnos, participando de buen grado no solo en las sesiones llevadas a cabo en el aula, sino también en las tareas que se les pidió que hicieran en sus casas, así como la buena predisposición mostrada para poder realizar las entrevistas personales, para lo que se utilizaron incluso horas de los patios.

Realmente es significativo señalar la motivación con la que abordaron las sesiones desde el primer momento, sintiéndose partícipes del proceso de enseñanza-aprendizaje, como demuestra el alto grado de participación en la primera de las sesiones de discusión en grupo, en la que se expusieron los resultados de la encuesta que se les pasó para tratar sus preferencias musicales y los hábitos de escucha, lo que permitió captar matices que ellos mismos aportaban que en una encuesta no se pueden percibir -y que consideramos necesario conocer para comprender su aprendizaje musical- lo que enriqueció enormemente nuestro punto de vista.

El pedirles propuestas de canciones para analizar en clase supuso otro momento importante (18 de los 20 alumnos trajeron a la siguiente sesión "sus canciones”), situándolos como protagonistas, incluso para elegir las tres que finalmente serían analizadas -lo que supuso un importante trabajo de carácter grupal para alcanzar el consenso-. 
Uno de los aspectos que más nos llamó la atención fue la actitud con la que abordaron el trabajo con los modelos elaborados para analizar las audiciones -tanto para la música actual como para la culta-. El hecho de establecer paralelismos entre ambos de manera constante supuso que el grado de interés, atención y participación con el que afrontaron las sesiones de audición fuera excelente, e incluso ellos mismos reconocían haber sentido curiosidad por los paralelismos existentes en las audiciones que se les presentaron de música culta anunciadas por el profesor, lo que les mantuvo realmente atentos -de una manera un tanto inusual-, lo que supone una mejora importante en el proceso de desarrollo de la capacidad de análisis auditivo.

Además, el trabajo realizado en el aula facilitó la comprensión de aquellos aspectos del lenguaje musical que tradicionalmente son más complicados de entender, como por ejemplo el papel que desempeña el acompañamiento armónico en el carácter de una melodía, o la influencia de los diferentes tipos de texturas en el resultado sonoro final.

\section{6.- CONCLUSIONES}

El trabajo resume una experiencia didáctica diseñada para dar respuesta a una serie de interrogantes planteados con el fin de introducir mejoras en el ámbito de la didáctica de la audición musical en la Educación Secundaria Obligatoria.

La revisión de la literatura existente sobre la influencia de la educación musical en la formación integral de los alumnos no hizo sino confirmar la importancia del desarrollo de las capacidades perceptivas en esta etapa educativa. Estamos convencidos de que debemos procurarles una audición más activa y en este sentido nos mostramos totalmente de acuerdo con Copland (1994, p. 35) cuando afirma que: "lo mismo si escuchamos a Mozart que a Duke Ellington, podremos hacer más honda nuestra comprensión de la música con sólo ser unos oyentes más conscientes y enterados, no alguien que se limita a escuchar, sino alguien que escucha algo".

Por otra parte, el hecho de elaborar una selección de obras de música culta que sean idóneas en términos didácticos para ser trabajadas con este tipo de alumnado nos permite, por una parte, actualizar el repertorio de música culta a utilizar y por otro, estar preparados para tender puentes entre este tipo de música y la que ellos suelen escuchar, lo cual enriquece el proceso de enseñanzaaprendizaje, pues como se ha demostrado la elección del repertorio influye en gran medida en las actitudes con que los alumnos afrontan las actividades.

Pensamos que la experiencia podría ser transferible a otros contextos, si bien no pretendemos generalizarla, por lo que hemos tratado de proporcionar descripciones del proceso seguido lo suficientemente minuciosas como para que el lector pueda decidir si los resultados obtenidos son aplicables a otras situaciones.

\section{7.- REFERENCIAS}

Aranguren, A. I. (2011). El consumo musical adolescente como fuente de estrategias didácticas. Eufonía, Didáctica de la música 53, 25-33.

Barceló, B. (1998). Psicología de la conducta musical en el niño. Palma de Mallorca: ICE Universidad de las Islas Baleares.

Bresler, L. (2006). Etnografía, fenomenología e investigación-acción en educación musical. En M. Díaz (coord.), Introducción a la investigación en educación musical (pp. 83-99). Madrid: Enclave Creativa Ediciones SL.

Copland, A. (1994). Cómo escuchar la música. Madrid: Fondo de Cultura Económica.

Gardner, H. (1995). Inteligencias múltiples. Buenos Aires: Paidós.

Lacárcel Moreno, J. (2003). Psicología de la música y emoción musical. Educatio Siglo XXI, 20.

Leiva, M. A., y Mates, E. M. (1998). Importancia de la educación musical en la formación integral del hombre. Música y Educación 33, 27-40.

Malbrán, S. (1996). Los atributos de la audición musical: Notas para su descripción. Eufonía: Didáctica de la música 2, 55-68.

Maneveau, G. (1993): Música y Educación. Madrid: Rialp. 
Palacios, F. (1997). Escuchar: 20 reflexiones sobre música y educación musical. Las Palmas de Gran Canaria: Fundación Orquesta Filarmónica de Gran Canaria.

Palacios, F. (2011). Hablar de escuchar: algunas reflexiones sobre música y educación musical. Vitoria-Gasteiz: Agruparte Producciones.

Paynter, J. (1999). Sonido y estructura. Madrid: Ediciones Akal.

Ramírez, C. (2006): Música, lenguaje y educación. La comunicación humana a través de la música en el proceso educativo. Valencia: Tirant lo Blanch.

Decreto 112/2007, de 20 de julio, del Consell, por el que se establece el currículo de la Educación Secundaria Obligatoria en la Comunitat Valenciana.

Willems, E. (2011). El oído musical. La preparación auditiva del niño. Barcelona: Paidós Educador.

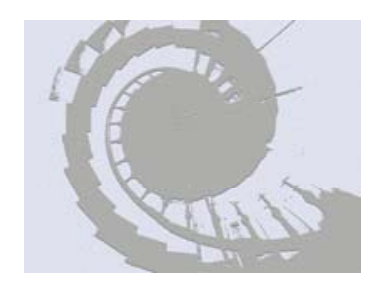

